\title{
Outcomes of the Laparoscopic Sleeve Gastrectomy in the Medicare Population
}

\author{
Brooke Bayham • Frank Greenway • Drake Bellanger
}

Published online: 22 August 2012

(C) Springer Science + Business Media, LLC 2012

Recently, we reported on a series of 529 patients who underwent the sleeve gastrectomy (SG) without experiencing a gastrointestinal leak [1]. The results indicate that the procedure is becoming more standardized and thus safer. The Centers for Medicare and Medicaid Services (CMS) approved coverage for the gastric bypass and the adjustable gastric band for individuals who meet the National Institutes of Health criteria for weight loss surgery in 2006. However, CMS has only recently issued a directive regarding Medicare reimbursement for SG. During this consideration, two concerns have arisen: (1) the limited number of SG surgeries reported in the Medicare age group and (2) the potential for a greater risk of complications occurring in the Medicare age group compared to younger adults. We reexamined our retrospective chart review of 766 laparoscopic SG patients and found 15 subjects who fell into the Medicare age group ( $\geq 65$ years of age). Here, we report on our results.

Of the 15 patients, there were 8 females and 7 males. All were white, and none had insurance coverage. The average preoperative weight and BMI were $124.5 \mathrm{~kg}$ and $43.3 \mathrm{~kg} / \mathrm{m}^{2}$, respectively. The average total body weight loss in the Medicare age group was significant, yet more modest than the younger age group ( $<65$ years of age) at 6 months and 1 year $(13.5$ and $24.1 \%$ versus $\sim 20$ and $\sim 29 \%$, respectively). All of the subjects in the Medicare age group had one or more comorbidities (primarily hypertension, hypercholesterolemia, and type 2 diabetes), and 11 $(73.3 \%)$ had three or more comorbidities. Some patients were able to discontinue their medications for cholesterol (60\%), diabetes (56\%), and hypertension (31\%) postoperatively, and many others were able to do dose reductions. Similarly, a study examining patients over 59 years found that 83 and $58 \%$ of patients resolved their T2D and HTN, respectively [2], and

B. Bayham $\cdot$ F. Greenway $(\bowtie) \cdot$ D. Bellanger

Outpatient Clinic, Pennington Biomedical Research Center,

6400 Perkins Road,

Baton Rouge, LA 70808, USA

e-mail: frank.greenway@pbrc.edu another retrospective review found that elderly patients ( $>70$ years) were able to reduce their medications by at least $50 \%$ postoperatively [3].

Only 2 of our 15 patients reported a complication, and both were minor. One patient had an elevated blood pressure postoperatively during their hospital stay, and the other reported nausea and vomiting within the first 6 weeks postoperatively. We had no reports of mortality in the Medicare age group associated with SG which is concurrent with other studies examining the same population [2-4]. The younger age group $(n=751)$ had 15 major complications, but none were reported in the Medicare age group.

Thus, although we only had a limited number of subjects in the Medicare age group, our data demonstrate the medical benefits of SG. Most subjects experienced improvement or resolution of their comorbid conditions, and the Medicare age group did not appear to be at an increased risk of major complications compared to the younger age group.

Conflict of Interest Pennington Biomedical Research Center received a grant from Ethicon Endosurgery, Inc., to fund the study. DB received funds from Ethicon Endosurgery, Inc., for lectures and service on speaker's bureau. BB and FG have no conflicts to declare.

\section{References}

1. Bellanger DE, Greenway FL. Laparoscopic sleeve gastrectomy, 529 cases without a leak: short-term results and technical considerations. Obes Surg. 2011;21(2):146-50.

2. Leivonen MK, Juuti A, Jaser N, et al. Laparoscopic sleeve gastrectomy in patients over 59 years: early recovery and 12-month followup. Obes Surg. 2011;21(8):1180-7.

3. Ramirez A, Roy M, Hidalgo JE, et al. Outcomes of bariatric surgery in patients $>70$ years old. Surg Obes Relat Dis. 2012;8:458-62.

4. O'Keefe KL, Kemmeter PR, Kemmeter KD. Bariatric surgery outcomes in patients aged 65 years and older at an American Society for Metabolic and Bariatric Surgery Center of Excellence. Obes Surg. 2010;20(9):1199-205. 